A C TA C H E I CA SCA N D I N A V ICA 8 (1954) $407-411$

\title{
Die Verschiedenheit der durch Amylasen spaltbaren Bindungen der Kartoffelstärke
}

\author{
JAKOB BLOM und BIRGIT SCHWARZ
}

Laboratorium der Tuborg-Brauereien, Kopenhagen, Dänemark

\begin{abstract}
Der Abbau von Kartoffelstärkekleister durch Pankreas-, Subtilisund Gersten-Amylasen wird durch gleichzeitige Messungen des Drehungs- und Reduktionsvermögens verfolgt. Die Änderung der Drehung per Mol gespaltener Bindungen, $[S p]_{D}$, ist verschieden für die verschiedenen Amylasen und abhängig vom Abbaugrad. Dieses Resultat lässt sich nur dadurch erklären, dass Stärke verschiedene durch Amylasen spaltbare Bindungen enthält.
\end{abstract}

Tn der vorigen Arbeit ${ }^{1}$ haben wir uns die Frage gestellt: "Welche Drehungs-

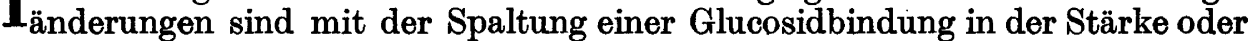
in Stärkeabbauprodukten verbunden, und zwar von Gleichgewichtszustand zu Gleichgewichtszustand gemessen." Die Änderung der Drehung per Mol gespaltener Bindungen haben wir mit $[\mathrm{Sp}]_{\mathrm{D}}$ bezeichnet. Versuche über die Säurehydrolyse von Kartoffelstärkekleister ergaben, dass [Sp] während der Hydrolyse nicht konstant ist. Von Punkt zu Punkt berechnet ist [Sp $]_{\mathfrak{D}}$ anfangs ca. $-160^{\circ}$ und fällt während des Abbaus, um bei einem Abbau von ca. $60 \%$ seinen minimalen Wert ca. $-280^{\circ}$ zu erreichen.

In der vorliegenden Arbeit bringen wir Resultate von Untersuchungen über den Abbau von Kartoffelstärkekleister durch Amylasen. Der Stärkeabbau wurde bei $40^{\circ}$ durchgeführt, und zwar bei pH 6-7 in Versuchen mit Pankreasamylase, bei pH 5,2 in Versuchen mit Subtilisamylase und Gerstenamylase. Aus dem Ansatz wurden in passenden Intervallen Proben entnommen, in denen die Enzymwirkung entweder durch Kochen oder durch Ansäuern auf pH 1-2 sistiert wurde. In den Proben wurden Reduktions- und Drehungsvermögen bestimmt. In einigen Serien wurde das Reduktionsvermögen mit einer Kupfermethode, in anderen Serien mit einer Jodmethode bestimmt. Das Drehungsvermögen ist erst nach einem Abbau von ca. $10 \%$ TM bestimmbar, da die Stärkelösung vorher nicht klar genug ist. Die Resultate sind graphisch dargestellt. Das Reduktionsvermögen, $\mathrm{R}$, in mmol reduzierender Zucker per $100,0 \mathrm{~g}$ Lösung berechnet, ist Abscisse; das Drehungsvermögen, $\alpha_{\text {korr }}$, auf 1 $\mathrm{dm}$-Rohr umgerechnet und mit der Dichte korrigiert, ist Ordinate.

Acta Chem. Scand. 8 (1954) No. 3 


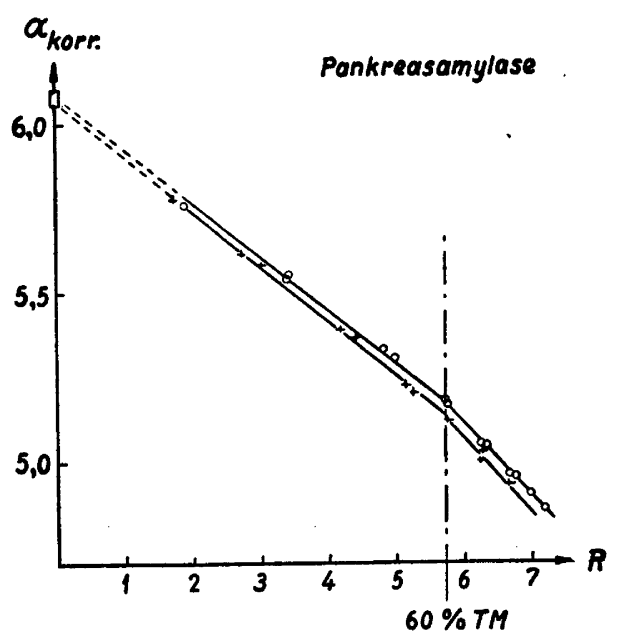

Figur 1. Abbau von Kartoffelstärke. Kleister $3,00 \%, p H=6-7, t=40^{\circ}$.

$R$ : mmol reduzierender Zucker per 100,0 $g$ Lösung,

+ Kupfermethode, o Jodmethode.

akorr: Drehung, korrigiert für Dichte, 1 dm-Rohr, D-Linie.

$[S p]_{\mathrm{D}}$ : Kupfermethode, bis $60 \%$ TM: $-164^{\circ}$; nach $60 \%$ TM: $-216^{\circ}$. Jodmethode, bis $60 \%$ TM: $-159^{\circ}$; nach $60 \%$ TM: $-215^{\circ}$.

Die Resultate der Versuche mit Pankreasamylase sind in Figur 1 dargestellt. Die beiden Methoden zur Bestimmung des Reduktionsvermögens geben Kurven, die etwas gegen einander verschoben sind. Es ist uns nicht möglich gewesen einen plausiblen Grund dafür zu finden. Bis zu einem Abbaugrad von ca. $60 \%$ TM, d.h. bis zu einer Spaltung von ca. $30 \%$ aller Bindungen der Stärke, liegen die Punkte auf einer Geraden. Nach $60 \%$ TM liegen die Punkte ebenfalls auf einer Geraden, die aber eine andere Neigung hat. Die Neigung der Geraden ist $[\mathrm{Sp}]_{\mathrm{D}} \cdot 10^{-3}$; sie wird nach der Methode der kleinsten Quadrate berechnet.

In Figur 2 und 3 sind die Resultate der Versuche mit Subtilisamylase und Gerstenamylase dargestellt. Im Gegensatz zu den Versuchen mit Pankreasamylase geben die beiden Methoden zur Bestimmung des Reduktionsvermögens Kurven, die sich decken, weshalb wir es für nottig gefunden haben, die Resultate getrennt wiederzugeben. Die linken Hälften der Figuren zeigen den Abbau durch Subtilisamylase, die rechten Hälften einen fortgesetzten Abbau durch Gerstenamylase, nachdem die Subtilisamylase den schnellen Teil ihres Angriffes durchgeführt hat und durch Kochen sistiert worden ist. Für jedes Enzym liegen die Punkte auf Geraden, deren Neigung und damit [Sp $]_{\mathrm{D}}$ verschieden ist.

In Tabelle 1 haben wir die $[S p]_{D}$-Werte zusammengestellt. Innerhalb der Fehlergrenzen sind die mittels der Kupfermethode und die mittels der Jodmethode gefundenen Werte paarweise gleich. Der Stärkeabbau durch Pankreasamylase gibt zwei $[\mathrm{Sp}]_{\mathrm{D}}$-Werte. Die Änderung tritt abrupt bei ca. $60 \%$ TM ein und zwar gleichzeitig mit der Überschreitung des achroischen Punktes und mit der früher gefundenen grossen Geschwindigkeitsabnahme ${ }^{2}$ des Stärkeabbaus. Der Abbau von Stärkekleister durch Subtilisamylase wird, wie früher gezeigt ${ }^{3}$, bei einem Abbaugrad von 35-40\% TM gehemmt; die Abbauprodukte werden durch Gerstenamylase schnell gespalten. Der $[\mathrm{Sp}]_{\mathbb{D}}$-Wert für den Abbau von Stärkekleister durch Subtilisamylase ist verschieden von dem 


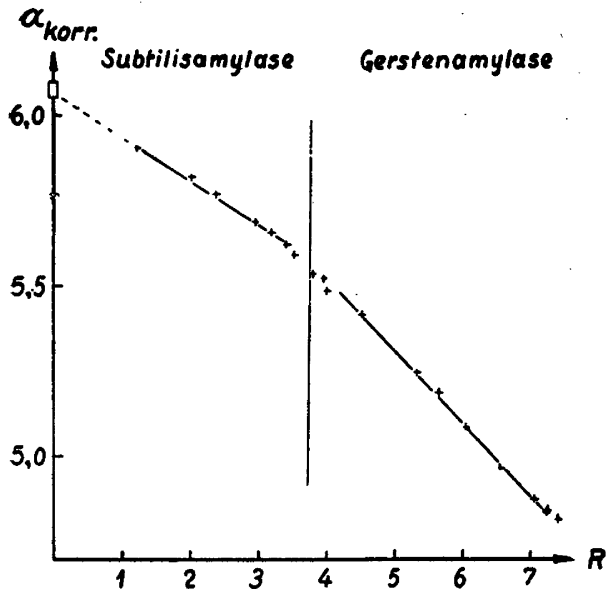

Figur 2. Abbau von Kartoffelstärke. Kleister $3,00 \%, p H=5,2, t=40^{\circ}$.

$R:$ mmol reduzierender Zucker per 100,0 g Lösung, Kupfermethode.

akors: Drehung, korrigiert für Dichte, $1 \mathrm{dm}$. Rohr, D-Linie.

$[S p]_{\mathrm{D}}$ : Subtilisamylase: $-129^{\circ}$. Gerstenamylase: $-205^{\circ}$.

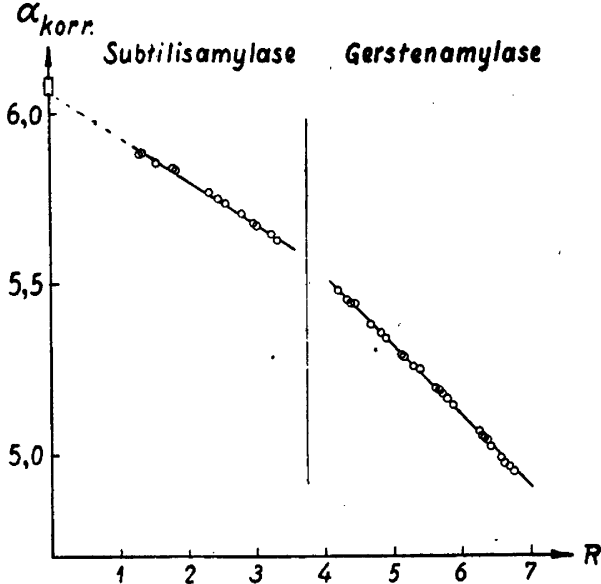

Figur 3. Abbau von Kartoffelstärke. Kleister $3,00 \%, p H=5,2, t=40^{\circ}$.

$R$ : mmol reduzierender Zucker per 100,0 g Lösung, Jodmethode.

akorr: Drehung, korrigiert für Dichte, $1 \mathrm{dm}$. Rohr, D-Linie.

$[S p]_{\mathrm{D}}$ : Subtilisamylase: $-129^{\circ}$.

Gerstenamylase: $-204^{\circ}$.

$[\mathrm{Sp}]_{\mathfrak{D}}$-Wert für den weiteren Abbau der hinterlassenen Spaltprodukte durch Gerstenamylase.

Beim enzymatischen Abbau von Kartoffelstärkekleister erhält man also verschiedene [Sp]/-Werte, die innerhalb gewisser Abbaugrenzen konstant sind. Nach unserer Ansicht kann dieses Resultat nur dadurch erklärt werden, dass die Stärke verschiedene durch. Amylasen spaltbare Bindungen enthält.

Nach der heute üblichen Auffassung enthält Stärke nur zwei Bindungs-

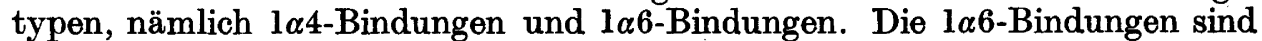
gegen Amylasen resistent; Isomaltose, 6-( $\alpha$-Glucosido $)$ - Glucose, wurde ja nach intensiver Behandlung von Stärke mit Amylasen isoliert 4,5. Hiernach können Amylasen nur 1a4-Bindungen spalten. Unter dieser Voraussetzung werden

Tabelle 1.

\begin{tabular}{|l|c|c|c|}
\hline \multicolumn{1}{|c|}{ Amylase } & $\begin{array}{c}\text { Abbau } \\
\text { \% TM }\end{array}$ & $\begin{array}{c}\text { Kupfermethode } \\
{[\mathrm{Sp}]_{\mathrm{D}}}\end{array}$ & $\begin{array}{c}\text { Jodmethode } \\
{[\mathrm{Sp}]_{\mathrm{D}}}\end{array}$ \\
\hline Pankreas- & $10-60$ & $-164^{\circ}$ & $-159^{\circ}$ \\
Pankreas- & $60-75$ & $-216^{\circ}$ & $-215^{\circ}$ \\
Subtilis- & $10-35$ & $-129^{\circ}$ & $-129^{\circ}$ \\
Gersten- nach Subtilis- & $\mathbf{4 5 - 7 0}$ & $-205^{\circ}$ & $-204^{\circ}$ \\
\hline
\end{tabular}

Acta Chem. Scand. 8 (1954) No. 3 
wir gezwungen anzunehmen, dass die Bezeichnung la4-Bindung nicht genügt, um einen Bindungstypus zu karakterisieren.

Die Anwesenheit von 1 1 5 -Bindungen in der Kartoffelstärke halten wir immer noch für möglich; die Anschauung von alternierenden 1a4- und la5Bindungen in einem Teil der Stärke ${ }^{1}$ haben wir jedoch verlassen.

\section{METHODIK}

Stärke. Kartoffelstärke. Superior Qualität.

Pankreasamylase. Aus frischem Schweinepankreas wird nach üblichen Methoden ein Dauerpräparat hergestellt.

Subtilisamylase. Eine 1.\%ige Lösung eines Trockenpräparates von Subtilisamylase wird mit Phosphatpuffer auf pH 7,0 eingestellt und 15 Minuten auf $70^{\circ}$ erwärmt.

Gerstenamylase. Ein Gerstenauszug wird durch Ansäuern ( $\mathrm{pH} 3,8,0^{\circ}, 16$ Stunden) von jeder Spur von $\alpha$-Amylase befreit. Durch Fällung mit Tannin wird ein Dauerpräparat hergestellt.

Bestimmung des Drehungsvermögens. Polarisationsapparat von Schmidt und Haensch, Monochromator, D-Linie, $4 \mathrm{dm}-\mathrm{Rohr}, 19^{\circ}-21^{\circ}$.

Bestimmung des Reduktionsvermögens. Kupfermethode: Kaliumkupferkarbonatmethode nach Blom-Rosted s. Jodmethode: Methode nach Willstätter-Schudel in der von uns beschriebenen Ausführung ${ }^{1}$.

Stärkekleister. $15,00 \mathrm{~g}$ trockene, aschenfreie Stärke werden in einem, mit zugehörigem Propeller tarierten, $500 \mathrm{ml}$ Weithals-Stehkolben abgewogen und durch Rühren mit einem schnell laufenden Motor in $400 \mathrm{ml}$ Wasser aufgeschlämmt. Ohne des Rühren zu unterbrechen, wird der Kolben in ein siedendes Wasserbad getaucht und nach 15 Minuten in ein Wasserbad von ca. $40^{\circ}$ überführt. Nach weiteren 15 Minuten wird das Rühren unterbrochen.

Abbau durch Pankreasamylase. a) (Oktober 1951). Der Kleister wird mit 10,0 ml $1 \mathrm{M}$ $\mathrm{KH}_{2} \mathrm{PO}_{4}, 10,0 \mathrm{ml} 1 M \mathrm{Na}_{2} \mathrm{HPO}_{4}, 10,0 \mathrm{ml} 1 M \mathrm{NaCl}$ und 1,0 bis $10,0 \mathrm{ml}$ einer 0,5-\%igen Lösung von Pankreasamylase versetzt; der Inhalt des Kolbens wird auf 500,0 g mit Wasser aufgewogen und eine Minute gerührt. Der Kolben wird verschlossen in ein Wasserbad von $40^{\circ}$ getaucht. Zu verschiedenen Zeitpunkten (15 Minuten bis 4 Stunden) werden Proben von ca. $100 \mathrm{~g}$ entnommen, die in verschlossenen Kolben 10-15 Minuten in einem siedenden Wasserbad erhitzt werden, um die Wirkung der Amylase zu sistieren. Nach Abkühlen werden die Lösungen durch $9 \mathrm{~cm}$ Filter, worauf ca. $75 \mathrm{mg}$ Kieselgur ange. bracht sind, filtriert. In den letzten $25 \mathrm{ml}$ der Filtrate werden Drehungsvermögen, Dichte und Reduktionsvermögen nach der Kupfermethode bestimmt.

b) (Juni 1952). Der Kleister wird mit 2,0 bis 30,0 ml einer 2-\%igen Lösung von Pankreasamylase versetzt und eine Minute gerührt. Der Kolben wird verschlossen in ein Wasserbed von $40^{\circ}$ getaucht. Zu verschiedenen Zeitpunkten (20 bis 160 Minuten) werden Proben von ca. $90 \mathrm{~g}$ entnommen und in tarierten Kolben, ca. $2 \mathrm{~g} 4 \mathrm{~N}$ HCl enthaltend, gegossen. Dadurch wird $\mathrm{pH}$ auf ca. 1 verschoben, um die Wirkung der Pankreasamylase zu sistieren. Durch Wiegen vor und nach den einzelnen Manipulationen wird genauer Kontakt mit der ursprünglichen Stärkemenge bewahrt. Nach Abkühlen und Filtrieren, wie unter a) beschrieben, werden in den letzten $25 \mathrm{ml}$ der Filtrate Drehungsvermögen, Dichte und Reduktionsvermögen nach der Jodmethode bestimmt.

Abbau durch Subtilisamylase. Der Abbau wird in derselben Weise wie unter Pankreasamylase beschrieben durchgeführt, nur werden in allen Versuchen als Puffer $15,0 \mathrm{ml} 1,2$ $M \mathrm{CH}_{3} \mathrm{COONa}$ und 5,0 ml 1,2 $\mathrm{M} \mathrm{CH}_{3} \mathrm{COOH}$ benutzt. Die Enzymmengen betragen 4,0 bis 15,0 ml einer 1.\%igen Lösung von Subtilisamylase, und der Abbau dauert höchstens 200 Minuten. (a) Januar 1951, b) Mai 1952.)

Abbau der von Subtilisamylase hinterlassenen Spaltprodukte durch Gerstenamylase. Der Kleister wird mit Acetatpuffer und Subtilisamylase und evt. einigen Tropfen Toluol versetzt und eine Minute gerührt. Der Kolben wird verschlossen in ein Wasserbad von $40^{\circ}$ getaucht. Nach passender Zeit (3 bis 18 Stunden) wird die Amylasewirkung durch Kochen sistiert. Während des ganzen Versuches wird durch Wiegen vor und nach den einzelnen Manipulationen genauer Kontakt mit der ursprünglichen Stärkemenge bewahrt. 
,

a) (Februar 1951). Eine Probe wird entnommen und der Rest des Kolbeninhalts mit Gerstenamylase (3 bis $20 \mathrm{mg}$ ) versetzt. Der Abbau wird wie unter Pankreasamylase a) beschrieben durchgeführt. Gesamtdauer bis zu 4 Stunden.

b) (April 1953). Die gesamte Portion des durch Subtilisamylase abgebauten Kleisters wird durch ein $15 \mathrm{~cm}$ Filter, worauf ca. $200 \mathrm{mg}$ Kieselgur angebracht sind, filtriert; die ersten $25 \mathrm{ml}$ des Filtrates werden verworfen. Eine Probe wird entnommen, der Rest der klaren Lösung wird mit Gerstenamylase $(8 \mathrm{bis} 16 \mathrm{mg}$ ) versetzt und der Kolben in ein Wasserbad von $40^{\circ}$ getaucht. Zu verschiedenen Zeitpunkten (10 bis 160 Minuten) werden Proben entnommen, worin die Wirkung der Amylase durch Ansäuern sistiert wird. Ohne Filtrieren werden die Lösungen wie unter Pankreasamylase b) beschrieben analysiert. Blindwert. Zwecks Bestimmung der Blindwerte, sowohl des Drehungs- als des Reduktionsvermögens, werden in allen Fällen vollständige Parallelversuche ohne Stärke durch. geführt.

\section{LITERATUR}

1. Blom, J. und Schwarz, B. Acta Chem. Scand. 6 (1952) 697.

2. Blom, J. und Schmith, T. Acta Chem. Scand. 1 (1947) 230.

3. Blom, J., Bak, A. und Braae, B. Hoppe-Seylers Z. physiol. Chem. 241 (1936) 273.

4. Ahlborg, K. und Myrbäck, K. Biochem. Z. 308 (1941) 187.

5. Montgomery, E. M., Weakley, F. B. und Hilbert, G. E. J. Am. Chem. Soc. 71 (1949) 1682.

6. Blom, J. und Rosted, C. O. Acta Chem. Scand. 1 (1947) 32.

Eingegangen am 28. November 1953. 